\title{
Three-dimensional photograph of electron tracks through a plastic scintillator
}

\author{
Mykhaylo Filipenko ${ }^{1, a}$, Timur Iskhakov ${ }^{2}$, Patrick Hufschmidt ${ }^{1}$, Gisela Anton ${ }^{1}$, Michael Campbell ${ }^{4}$, \\ Thomas Gleixner $^{1}$, Gerd Leuchs $^{2}$, Timo Tick ${ }^{4}$, John Vallerga ${ }^{3}$, Michael Wagenpfeil ${ }^{1}$, Thilo Michel ${ }^{1}$ \\ ${ }^{1}$ Erlangen Centre for Astroparticle Physics, University of Erlangen-Nuremberg, Erwin-Rommel-Straße 1, 91058 Erlangen, Germany \\ ${ }^{2}$ Max-Planck Institute for the Science of Light, Günther-Scharowsky-Straße 1/Bau 24, 91058 Erlangen, Germany \\ ${ }^{3}$ Experimental Astrophysics Group, Space Science Laboratory, University of California, Berkeley, CA 94720, USA \\ ${ }^{4}$ European Organization for Nuclear Research, CERN, 1211 Geneve 23, Switzerland
}

Received: 27 April 2014 / Accepted: 14 October 2014 / Published online: 11 November 2014

(C) The Author(s) 2014. This article is published with open access at Springerlink.com

\begin{abstract}
The reconstruction of particle trajectories makes it possible to distinguish between different types of charged particles. In high-energy physics, where trajectories are rather long (several meters), large size trackers must be used to achieve sufficient position resolution. However, in lowbackground experiments like the search for neutrinoless double beta decay, tracks are rather short (some mm to several $\mathrm{cm}$, depending on the detector in use) and three-dimensional trajectories could only be resolved in gaseous time-projection chambers so far. For detectors of a large volume of around one cubic meter (large in the scope of neutrinoless double beta search) and therefore large drift distances (several decimeters to $1 \mathrm{~m}$ ), this technique is limited by diffusion and repulsion of charge carriers. In this work we present a "proof-of-principle" experiment for a new method of the three-dimensional tracking of charged particles by scintillation light: we used a setup consisting of a scintillator, mirrors, lenses, and a novel imaging device (the hybrid photon detector) in order to image two projections of electron tracks through the scintillator. We took data at the T-22 beamline at DESY with relativistic electrons with a kinetic energy of $5 \mathrm{GeV}$ and from this data successfully reconstructed their three-dimensional propagation path in the scintillator. With our setup we achieved a position resolution in the range of $170-248 \mu \mathrm{m}$.
\end{abstract}

\section{Introduction}

Since the beginning of the 20th century tracking detectors have played an outstanding role for discoveries in experimental particle and nuclear physics. After the first cloud

\footnotetext{
${ }^{a}$ e-mail: mykhaylo.filipenko@physik.uni-erlangen.de
}

chamber had been built by Charles Wilson in 1911, it was possible to observe tracks of charged particles [1]. By applying a magnetic field and analyzing the trajectories one was able to distinguish between electrons and positrons, which were discovered with a cloud chamber in 1932 [2]. With technological progress ongoing the cloud chamber was replaced with the bubble chamber [1] and the spark or wire chamber [3] which allowed higher event rates, better position resolution and an automated electrical data read-out. This technology was developed further to the gas-filled multi-wire proportional chamber [3] and the gas-filled time-projection chamber [4], which made reconstruction of trajectories in three dimensions possible.

After the advancement in semiconductor technology silicon strip detectors $[5,6]$ were developed and used for tracking applications. During the past ten years hybrid active pixelated semiconductor detectors are on the rise and expected to achieve a position resolution down to several $\mu \mathrm{m}$ for vertex tracking applications [7,8]. Besides these technologies, nuclear emulsions play an important role as a tracking detector in OPERA [9] and scintillating fiber trackers are discussed as a replacement for the downstream tracker in the $\mathrm{LHCb}$ upgrade [10].

Although with modern tracking detectors a very good position resolution (down to several micrometers) can be achieved for the detection of high-energy particles $\left(E_{\mathrm{kin}}>\right.$ $1 \mathrm{GeV}$ ), in the case of low-background experiments like the direct detection of dark matter or the neutrinoless double beta decay the same technologies cannot be used in the usual manner due to the specific requirements of these experiments. Since the main motivation behind this work is the search for neutrinoless double beta decay [11], the requirements of this specific research goal are discussed below. 
In this hypothetical decay two electrons are supposed to be emitted from a nucleus with a sum kinetic energy ranging from about $0.9 \mathrm{MeV}$ to about $4.8 \mathrm{MeV}$, depending on the nuclide of choice. In a neuntrinoless double beta decay the energy of both electrons has to be measured precisely to distinguish it from the neutrino-accompanied double beta decay. ${ }^{1}$ Usually in such experiments the decaying material is the sensitive detector volume because of two reasons: (a) The expected event rate is very low (smaller than 1 event per $100 \mathrm{~kg}$ of enriched material per year [12]) and therefore a large mass is required (with thin foils it is hard to achieve a high mass). (b) Electrons with energies on the MeV scale are easily scattered and therefore lose a significant part of their energy already on very small distances. Therefore, it is convenient to detect the electrons at their point of emission to avoid these losses.

In neutrinoless double beta decay experiments the main problem is the reduction of background (like beta decay events, Compton electrons, photoelectrons, $\alpha$-particles) which can produce false positive events. Tracking might be a valuable tool to identify such events and sort them out since the topological structure of the trajectory of the two decay electrons (from a neutrinoless double beta decay) is different from other ionizing radiation. On Fig. 1 a simulated electron with a kinetic energy of $2.8 \mathrm{MeV}$ ( $Q$ value of ${ }^{116} \mathrm{Cd}$, a neutrinoless double beta nuclide) and a simulated neutrinoless double beta event as could be measured in a cadmium-telluride Timepix detector are shown: in this case these two event types can be distinguished by the eye but also in more sophisticated cases methods like artificial neural networks can be used to identify different types of ionizing radiation [14-16].

The CSDA range (approximation to the average track length) of electrons with a low kinetic energy $(0.9 \mathrm{MeV}$ to $4.8 \mathrm{MeV}$ ) in solid or liquid materials that could be used for a neutrinoless double beta experiment is at most a few millimeters. As a consequence, they are usually completely stopped within one detector segment, such as one pixelated semiconductor sensor layer or one scintillator block, and therefore a track reconstruction in the manner of vertex tracking (where the position information from several tracking planes is combined) is not possible.

Nonetheless, in the future, tracking in neutrinoless double beta experiments could be performed by a pixelated readout of the charge signal with high granularity. It means that secondary electrons which were released in a gas or semiconductor material by the primary particle are drifted in an electric field to the collecting electrodes. Conductive wires (in TPCs) or active pixel detectors (like the Timepix) collect

\footnotetext{
${ }^{1}$ During a neutrino-accompanied double beta decay, the $Q$ value is distributed between two electrons and two anti-neutrinos, wherefore the sum energy of the two electrons is a broad distribution from 0 to the $Q$ value of the decay.
}

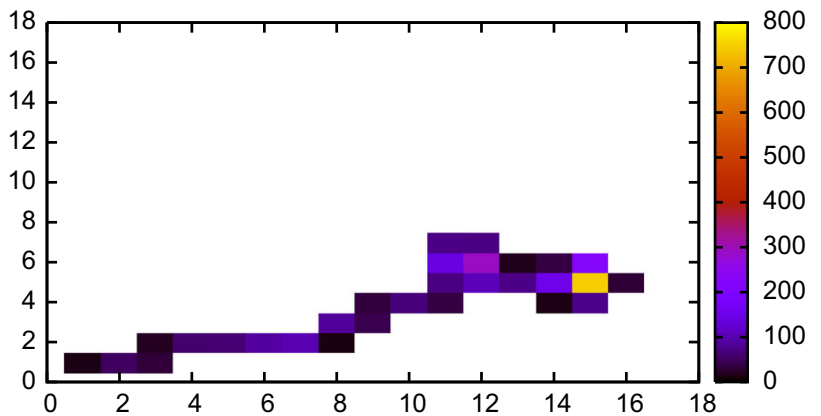

(a)

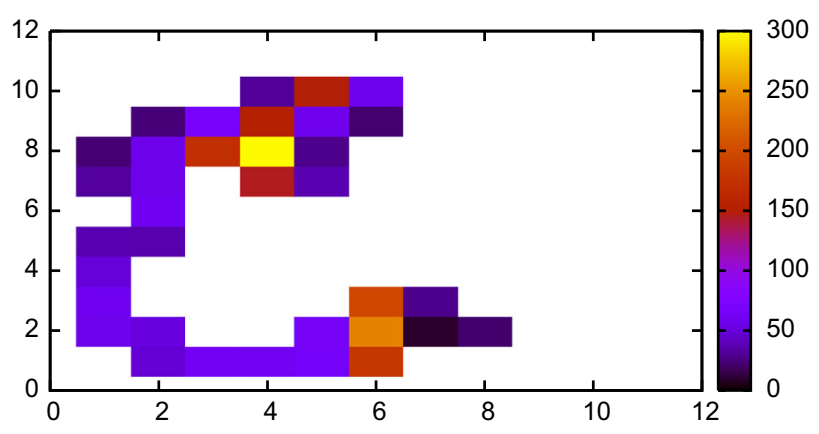

(b)

Fig. 1 Example of a simulated single electron (a) and neutrinoless double beta decay event (b) with $2.8 \mathrm{MeV}$ kinetic energy propagating through a cadmium-telluride sensor as could be detected with a Timepix-ASIC. The size of a pixel is $110 \mu \mathrm{m}$. The length of a track is $\approx 1.5 \mathrm{~mm}$. The color denotes the energy deposition per pixel in $\mathrm{keV}$

the released electrons and generate a signal by charge collection or the induction of currents. In micro pattern gaseous detectors, the electrons are drifted towards micromegas structures or GEM-foils. The transversal coordinates perpendicular to the drift direction can be measured by using at least two read-out planes (wire planes in TPCs) or segmentation of the collecting structure in pixels. In gaseous detectors, the drift time of the electrons is measured to reconstruct the drift distance ( $z$ coordinate) [16].

This method has the disadvantage that the position resolution is limited by the diffusion ${ }^{2}$ of charge carriers on their way through the sensor material (gas, semiconductor). This limitation makes it difficult to obtain a sufficient position resolution in a detector with large drift distance. For common materials (like liquid xenon) in use for neutrinoless double beta experiments the diffusion radius (the spread of the charge cloud in the plane perpendicular to the drift direction, when it arrives in the read-out plane) is larger than the

\footnotetext{
$\overline{2}$ Besides diffusion, also the repulsion of charge carriers contributes to the spread of the charge cloud on its way through the sensor. Here, we use the term diffusion as a roundup for diffusion, repulsion, and other possible effects that contribute to the spread of the charge cloud on its way through the sensor to the readout structure.
} 
primary particle's track length after the drift through the sensitive full volume (if the drift distance exceeds several $\mathrm{cm}$ ), which makes it impossible to resolve tracks in a large sensitive volume. ${ }^{3}$

This work presents a different approach for the measurement of three-dimensional trajectories of charged particles. The goal is to overcome the diffusion limitation. One possible way to do so is to use the scintillation signal instead of the ionization signal. Scintillation photons are created along the track and do not diffuse on their way through the sensor from the point of origin to the plane where they are detected. One can take advantage of this fact and reconstruct the three-dimensional particle trajectory from multiple twodimensional projections of the track imaged with a pixelated single photon detector. This publication presents a "proof-ofprinciple" demonstration of this method. We used a plastic scintillator, basic optical components (mirrors and lenses), and the hybrid photon detector, as explained in the next subsection.

\subsection{The hybrid photon detector}

The hybrid photon detector (HPD) is a first-of-its-kind detector that combines high spatial and temporal resolution for the detection of single optical photons [18]. The detector vacuum tube consists of a bi-alkali photocathode, which has a highest quantum efficiency of about $20 \%$ in the blue's/violet spectral range $(390 \mathrm{~nm})$. Underneath the photocathode there are two microchannel plates (MCPs) in a chevron configuration. Beneath those are four Timepix-ASICs [21] arranged in a $2 \times 2$ layout $(512 \times 512$ pixels). The tube is sealed under a vacuum pressure of $10^{-10} \mathrm{mbar}$. Photoelectrons which are released from the photocathode by optical photons are multiplied in an avalanche-cascade process in the MCPs. The $\mathrm{MCP}$ acts as a multi-channel photomultiplier. Therefore, one incident photon on the cathode results in an avalanche of about $10^{5}$ to $10^{6}$ electrons on the Timepix-ASIC electrodes with the MCPs retaining the information of the input location in the charge cloud centroid. The overall voltage difference between the photocathode and the Timepix is $2.4 \mathrm{kV}$. The Timepix-ASIC is at ground potential whereas the photocathode is at $-2.4 \mathrm{kV}$. Because the anode is pixelated, many photons can be detected concurrently. Depending on the mode of operation the timing resolution can be as good as $10 \mathrm{~ns}$ and the position resolution as good as $6 \mu \mathrm{m}$. The sensitive area is $2.8 \mathrm{~cm} \times 2.8 \mathrm{~cm}$. A photograph of the detector is shown in Fig. 2. Details on the HPD can be found in [18].

One Timepix-ASIC has $256 \times 256$ pixels with a pixel pitch of $55 \mu \mathrm{m}$. Each pixel has its own input electrode, connected to an analog circuitry with pre-amplifier and discriminator,

\footnotetext{
3 Tracks from some bottom part (close enough to the read-out plane) could be resolved but not from the full volume, which is the goal.
}

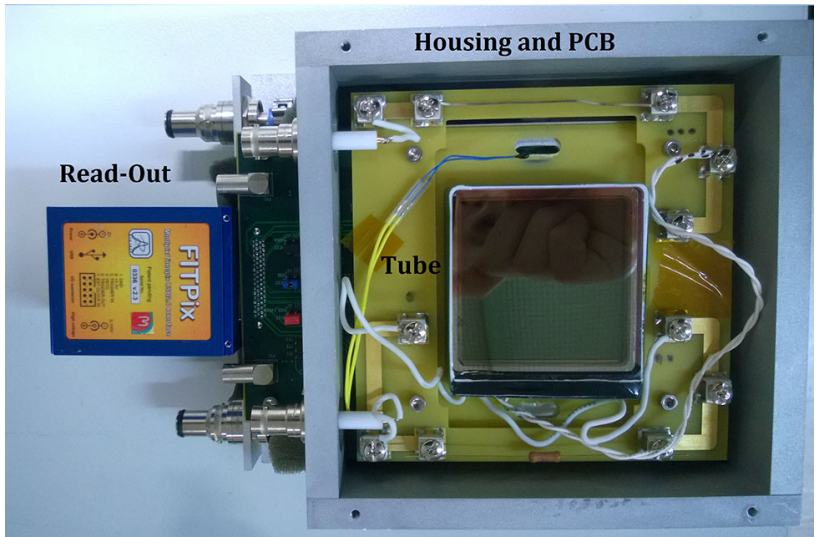

Fig. 2 A photograph of the hybrid photo detector (HPD) connected to a FitPix read-out [19]

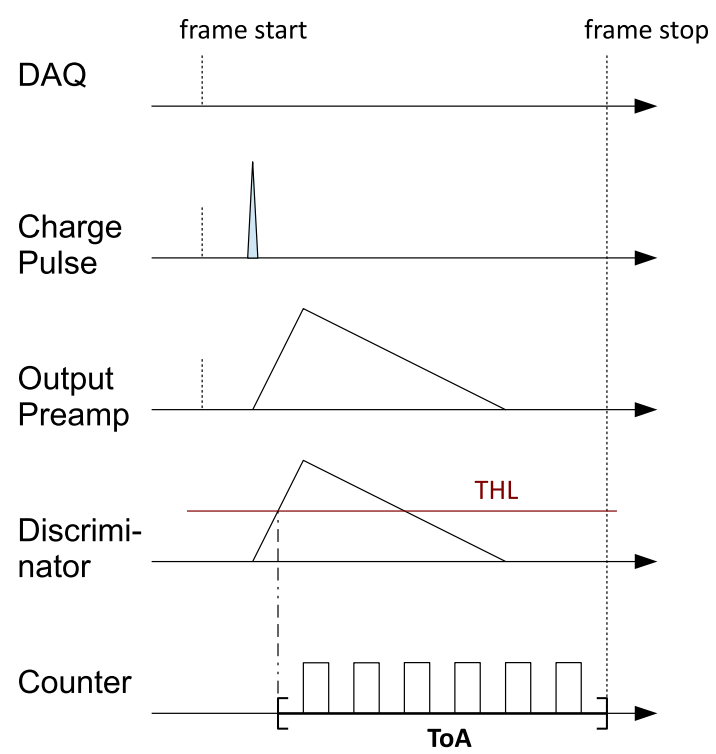

Fig. 3 The operation of the Timepix in the "time-of-arrival" (ToA) mode. After the charge pulse has been amplified and converted to a voltage pulse, it is discriminated against a threshold level (THL). The ToA is the number of clock cycles between the first time the voltage pulse rises over the THL and the end of the frame

and a digital electronics circuitry. In each pixel the charge is collected and converted into a voltage pulse. This is carried out by a Krummernacher type pre-amplifier. The voltage pulse in each pixel is discriminated against a global threshold which is equivalent to approximately $1,000 \mathrm{e}^{-}$. From this point on, the processing in each pixel is digital. It operates in three different ways but in this work only the so-called ToA mode was employed.

The ToA mode is illustrated in Fig. 3. When the voltage pulse rises above the threshold a digital register starts counting clock cycles until the end of the frame. The number of counted cycles is called ToA. This way, each triggered pixel gets a time stamp wherefore the ToA provides an absolute timing information for every pixel. A frame can have a fixed 
frame-time or be opened and closed by an external trigger (timing gate). The fastest possible clock frequency is about $100 \mathrm{MHz}$ wherefore every counted clock cycle corresponds to about $10 \mathrm{~ns}$. Details as regards the Timepix-ASIC can be found in [21].

\section{Experimental setup}

The main idea of the experiment was to reconstruct a three-dimensional particle trajectory from multiple twodimensional projections. When a charged particle propagates through a scintillator, scintillation photons are emitted isotropically along the track after relaxation of excited states. An optical system can be used to collect the isotropically emitted light for imaging. In the easiest possible scenario just one single lens and one detector can be used to create a two-dimensional image. If the same scintillator is imaged from multiple perspectives the information can be used to reconstruct a three-dimensional trajectory.

For a "proof-of-principle" demonstration of this method we used a setup as illustrated on Fig. 4. A cubic plastic scintillator (Bicron BC-408) with dimensions of $4 \mathrm{~mm} \times 4 \mathrm{~mm} \times 4$ $\mathrm{mm}$ was imaged from two orthogonal directions. The scintillation photons have a wavelength of about $425 \mathrm{~nm}$. We used two similar Thorlabs LB1761-A bi-convex lenses made of N-BK7 with a focal length of $f=25.4 \mathrm{~mm}$ and a diameter of $d=25.4 \mathrm{~mm}$. Two mirrors (Thorlabs BB1-E01) with a diameter of $25.4 \mathrm{~mm}$ were employed to adjust the optical path. One side of the scintillator was imaged on the top half

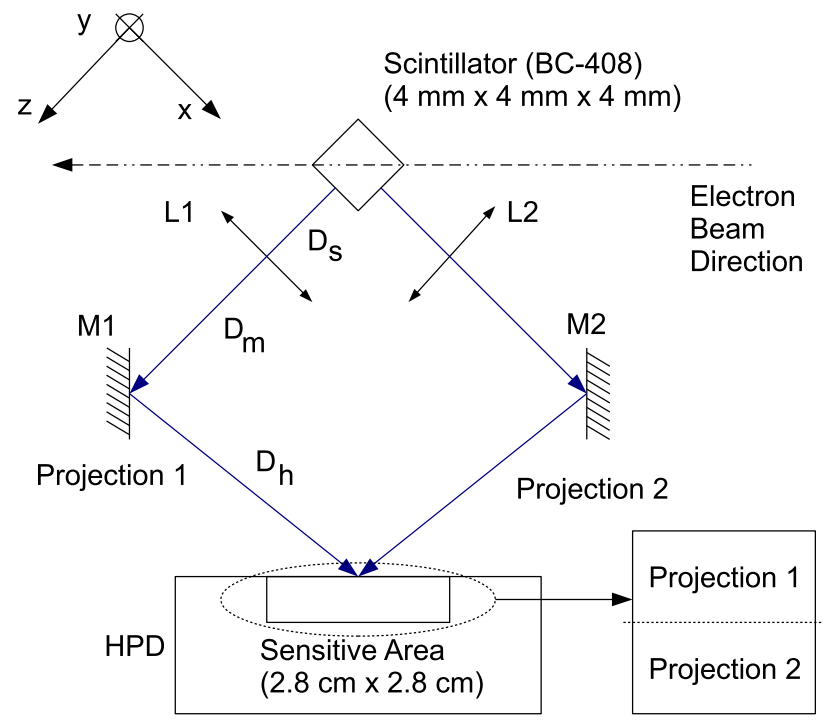

Fig. 4 Schematic view of the setup used for the measurements. It consists of a plastic scintillator, two lenses (L1, L2), two mirrors (M1, M2) and the HPD. One projection of the electron track in the scintillator is imaged on the top half of the HPD and the other on the bottom half. $D_{s}=34 \mathrm{~mm}, D_{m}=25 \mathrm{~mm}, D_{h}=77 \mathrm{~mm}$ of the HPD and one side on the bottom half. We adjusted the optical setup with a laser by imaging two sides of the scintillator on a paper screen held in front of the photocathode. We did not take an actual image with the camera to prevent the camera from taking any damage due to overillumination with the laser.

The distances between the optical elements were $D_{\mathrm{s}}=$ $34 \mathrm{~mm}, D_{\mathrm{m}}=25 \mathrm{~mm}$ and $D_{\mathrm{h}}=77 \mathrm{~mm}$. With this geometry we calculated a magnification of 3.3 and a light detection efficiency of about $1.5 \%$ per view (perspective) per image, taking into account the geometrical acceptance, the losses in the optical elements, and the absolute quantum efficiency of the HPD. Although the scheme suggests that the angles were $45^{\circ}$, in the real setup the angles were about $4^{\circ}$ off. With our geometry, the focal point spread function (or blur circle) $\sigma_{f}$ at a distance $d_{f}$ from the focal plane can be estimated as $\sigma_{f} \approx d_{f}$. It was calculated as described in [22]. Therefore, we expect to see sharp tracks only from an inner part of the scintillator around the focal plane $(500 \mu \mathrm{m}$ to each side of the focal plane).

We used two layers to shield the setup from external optical photons: the inner layer is a dark paper board from Thorlabs; the outer layer are walls made of $5 \mathrm{~mm}$ thick black plastic. A photograph of the setup is shown in Fig. 5. The dark rate of the HPD was 2,000 counts per second, which corresponds to the intrinsic dark rate of the detector.

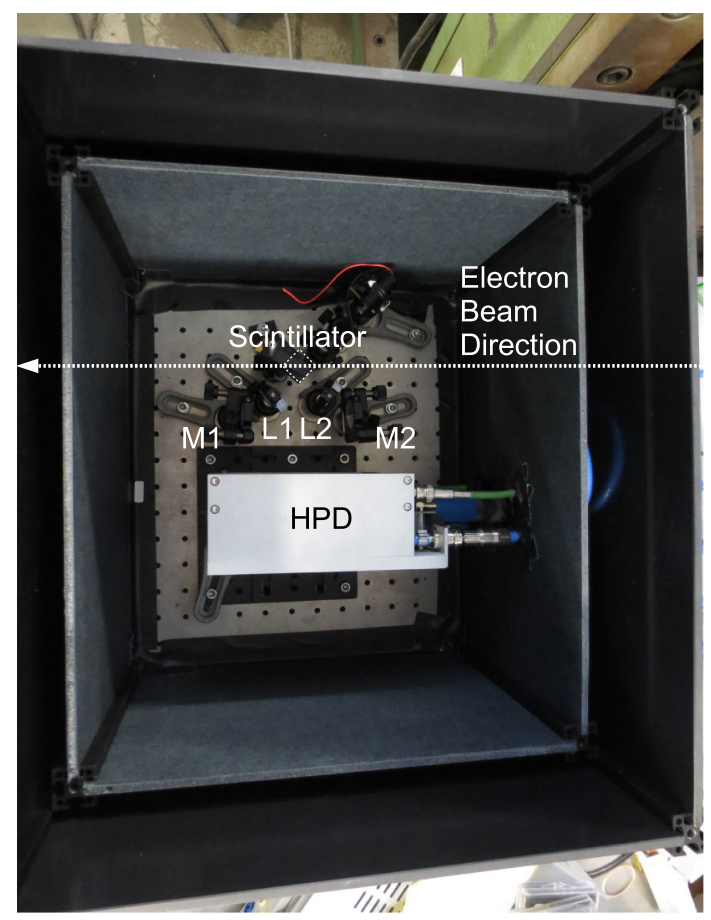

Fig. 5 A photograph of the setup used for the measurements. The detector and the optics were shielded by two layers (black plastic, dark paper board) from external optical photons. The beam direction is shown in the photograph 
(a)

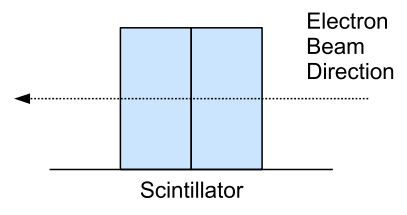

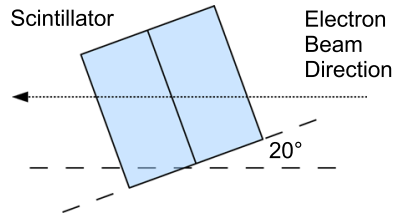

(b)
Fig. 6 The direction of the electron beam through the scintillator

We used the setup at the DESY testbeam T-22 where the scintillator was traversed by electrons with a kinetic energy of $5 \mathrm{GeV}$. Electrons of this energy can be regarded as minimally ionizing. Most of the time their trajectories through the scintillator are straight lines. Concerning the stopping power of the plastic scintillator at this energy (about $2.45 \frac{\mathrm{MeV}}{\mathrm{cm}^{2} \mathrm{~g}}$ ), we expect about 130 detected photons per view (projection) per image. The flux density of electrons was about $1,000 \frac{e^{-}}{\mathrm{s} \cdot \mathrm{cm}^{2}}$.

The maximum digital counter value in the Timepix ToA mode is 11,810 and as we used a clock of $10 \mathrm{MHz}(100 \mathrm{~ns}$ per clock cycle), the maximum frame integration time that could be used to avoid events with a maximum ToA value of 11,810 (and therefore with no useful) was $1 \mathrm{~ms}^{4}$ Since the read-out speed of the data acquisition system is limited, we could only start a frame every $0.1 \mathrm{~s}$. To avoid dead-time in-between and increase statistics (the number of measured events), we used the trigger signal from the beam monitor (two crossed scintillator panels with a PMT attached) for the frame-stop signal in order to have a higher probability to see one electron during one frame. However, as turned out later during the data analysis, our trigger system had a malfunction during the experiment and a large part of the data has to be rejected since the ToA value of the triggered pixels was 11,810 . Therefore, the actual frame-time fluctuated a lot.

We took data at two different orientations between the scintillator and the beam. In first case the beam transversed the scintillator from one edge of the scintillator to the other as shown in Figs. 4 and 6a. In the second case the setup was titled by $20^{\circ}$ with respect to the beam axis (Fig. 6b).

\section{Data analysis}

A typical frame contains either a track (as in Fig. 7a) as expected or some (randomly seeming) hits (Fig. 7b). Such frames happen if the electrons path through the scintillator was out of the optical focus, or possibly the trigger gate closed without any electron passing through the scintillator. This was possible since the sensitive area of the beam monitor

\footnotetext{
${ }^{4}$ If the ToA in the triggered pixels of an event is 11,810 , the pixel could have been triggered at any time between $11,810 \cdot 100 \mathrm{~ns}(\approx 1 \mathrm{~ms})$ and the end of the frame.
}

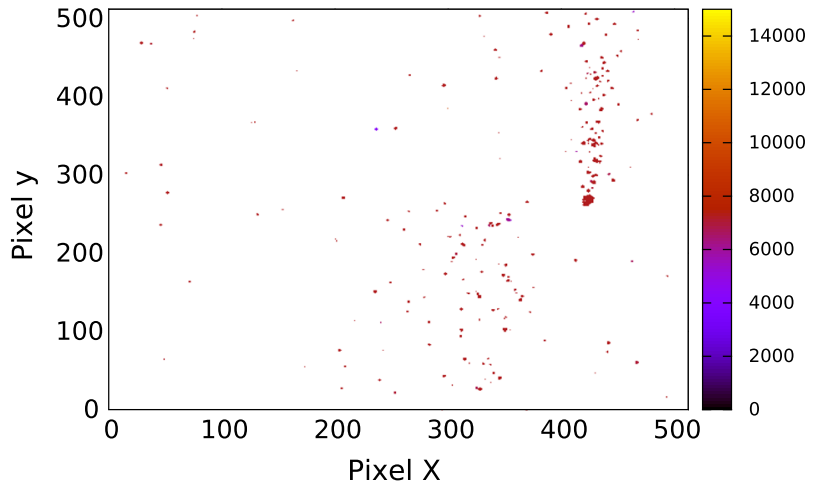

(a)

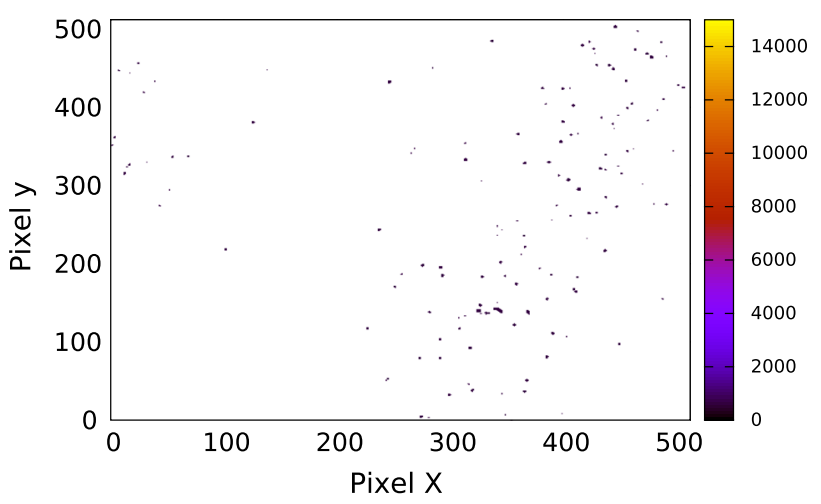

(b)

Fig. 7 Example of two typical frames: one with a track (a) and one with noise only (b). The color bar indicates the ToA measured in each triggered pixel

was larger than the cross section area of the scintillator in the setup.

Consequently, the first step was a frame selection due to the following criteria: First, we removed frames where less than 400 pixels were triggered. About 130 photons were expected per view (projection) per image and due to the electron avalanche of MCP amplification impinging on the Timepix ASIC, one photon usually triggers three to five pixels (resulting in about 780-1,300 triggered pixels for a proper event).

Secondly, for each frame all coincident hits registered with ToA values between 1 and the maximum counter value of 11,810 clock ticks were extracted. The condition for coincidence was that their ToA values differ by less than three clock ticks, corresponding to the time resolution of the system. These coincident hits were regarded to stem most probably from the same electron passage. Figure 8 shows an exemplary ToA spectrum integrated over 44,564 frames. For example, all hits at 1,930 clock cycles most probably stem from one electron trajectory.

During this evaluation it turned out that the time stamp of most events was 11,810 clock counts instead of three clock 


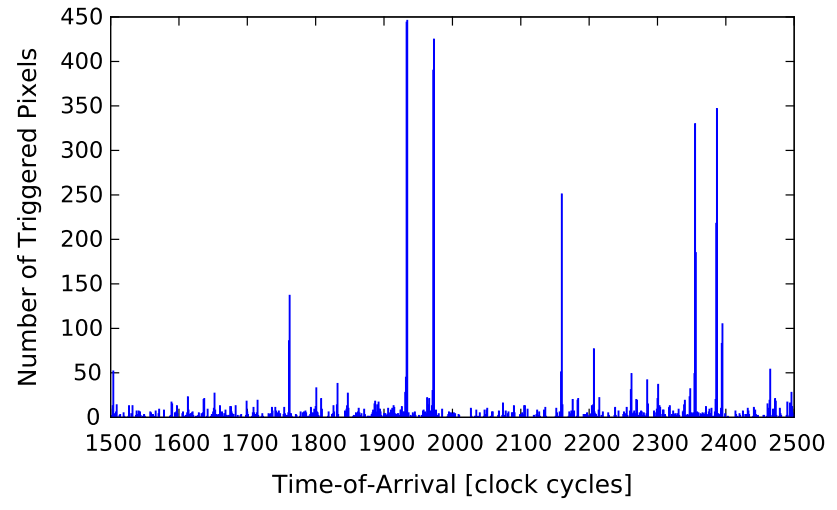

Fig. 8 Exemplary part of the ToA spectrum. Each peak belongs to the coincident detection of several photons; thus, to one electron track

counts as expected with the delay to the trigger. Therefore, we have to conclude an unexpected misbehavior occurred in the trigger system and the events between one clock cycle and 11,810 clock cycles accidentally appeared at a particular time in the frame. Nevertheless, since the triggered pixels appeared in the same frame and have common ToA values, it is very likely that they belong to the same event.

Next we performed a reduced Hough transform [23] of the frame for each projection, i.e. we calculated the angle between the $x$ axis and the connecting line of any two triggered pixels in each frame and sorted the angles into a histogram. A typical reduced Hough transform for one perspective of a frame containing a track (Fig. 9a) is shown in Fig. $9 \mathrm{~b}$ with the corresponding frame; a "bad frame" is shown in Fig. 9c with the corresponding reduced Hough transform (Fig. 9d). If a straight line appears in the projection, a clear peak can be seen in the reduced Hough transform. We integrated in an interval of $\left[-15^{\circ} ;+15^{\circ}\right]$ around the bin with the highest bin count and divided this integral by the integral of the remaining histogram. If the ratio was larger than 1 for both projections, the event was regarded in the further analysis, otherwise it was rejected. After these three frame selection steps the resulting number of trajectories was 26 starting from a total of 611 events with meaningful ToA value $(<11,810$ clock cycles) and a total of 44,564 frames. As discussed later, the frame selection probably lead to a selection of tracks in an interval of $\approx 0.25-0.6 \mathrm{~mm}$ around the focal plane.

For the three-dimensional trajectory reconstruction we "sliced" the two-dimensional images along the $y$ axis, parallel to the $x-/ z$-axis as shown in Fig. 10a. We obtained 512 $y$-slices in total; $256 y$-slices for the $x y$ plane and 256 slices for the $z y$ plane. The histograms of two single slices are shown in Fig. 10b. The average $x$-/z-position in every slice is determined by the mean value of a Gaussian fitted to each histogram. The fit is performed with the maximum likelihood method as the number of hits per slice is very low. If

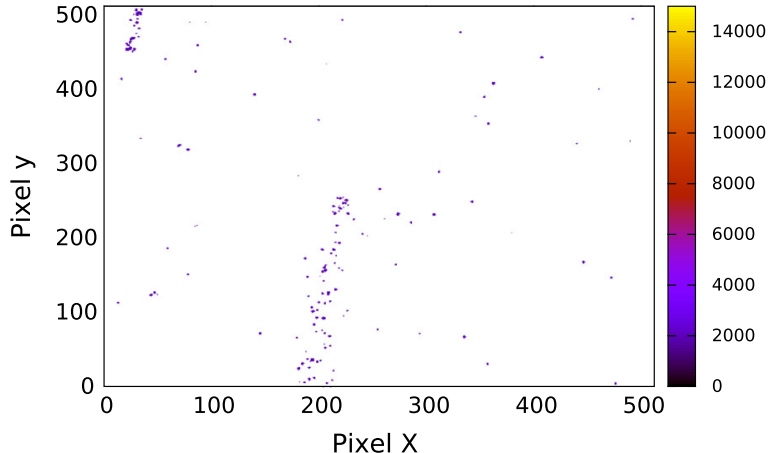

(a)

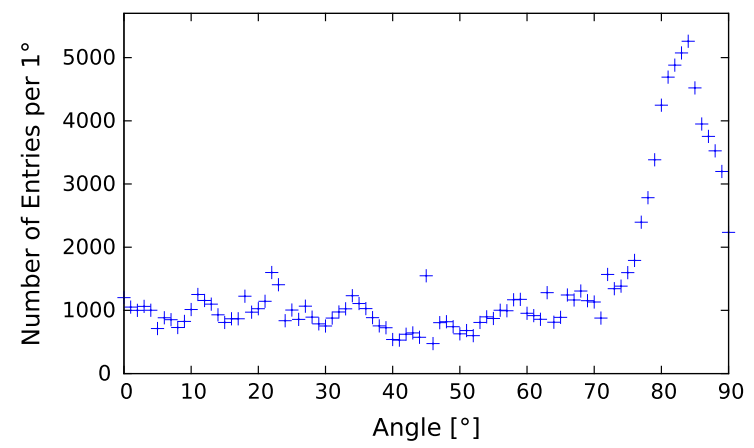

(b)

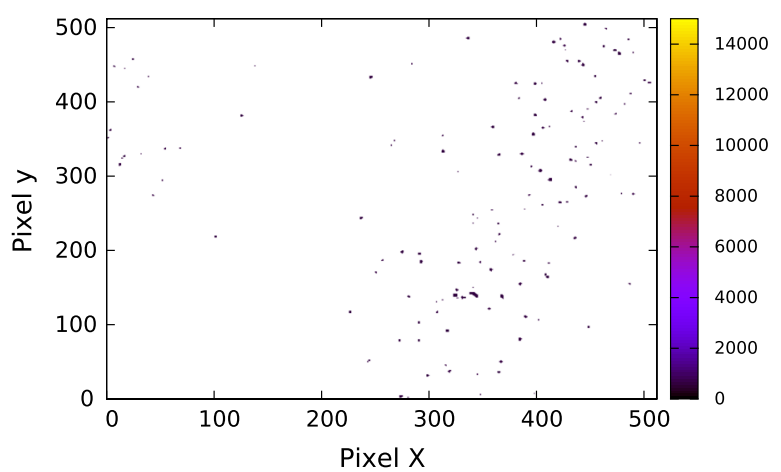

(c)

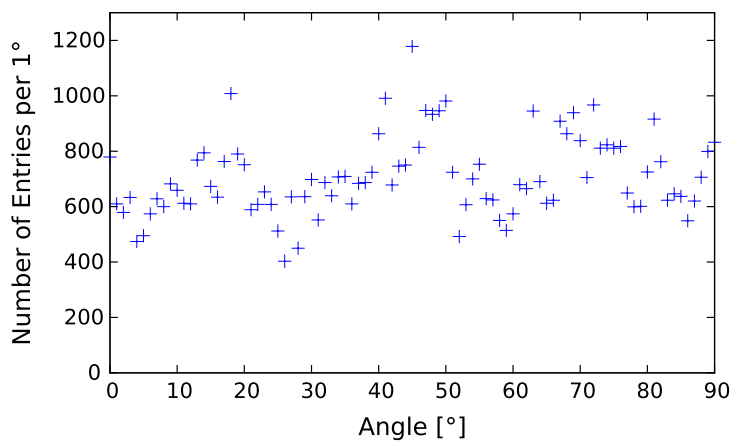

(d)

Fig. 9 Examples of images (a, c) and their reduced Hough transforms $(\mathbf{b}, \mathbf{d})$ in the $x y$ plane. If a track is in the plateau, peaks which belong to the direction of the track and a clear minimum appear in the transform. In the opposite case the distribution is flat. The color bar indicates the ToA measured in each triggered pixel 


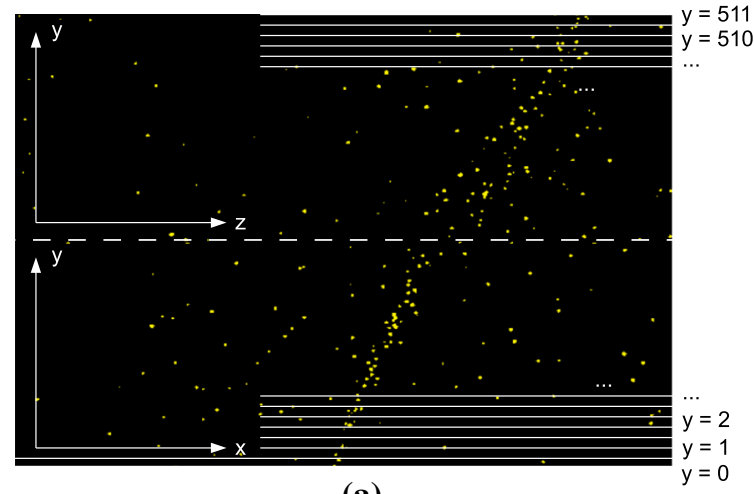

(a)
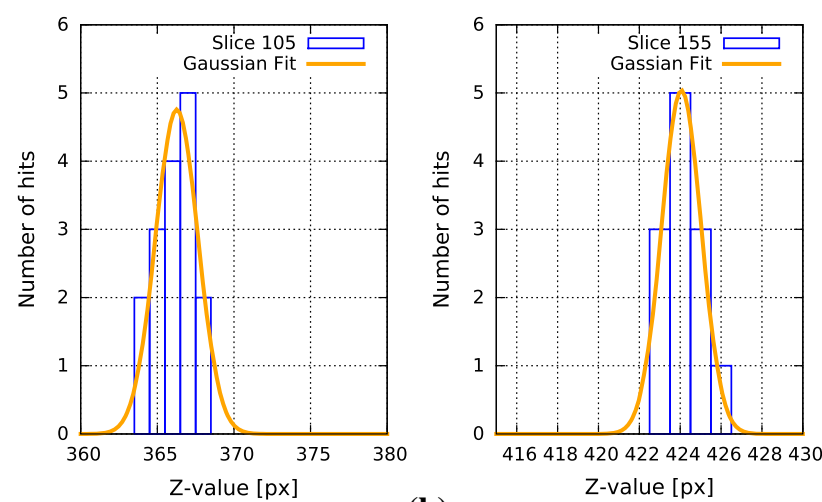

(b)

Fig. 10 An illustration of the slicing procedure. Each frame is divided into slices parallel to the $x$-/z-axis (a). In each slice the mean $x-/ z$ position is determined by a fit with a Gaussian. For each slice we obtain several histogram as in (b). The histograms shown here are summed over three consecutive slices for better statistics

the number of hits in the slice is zero, the slice is ignored. If the number of hits in the slice is smaller than four, the average $x$-position is used instead of the fit since in these cases the fit failed frequently.

The result of this procedure is shown in Fig. 11b. In this plot, the calculated $x$ value (on the ordinate) is plotted for every $y$-slice (on the abscissa). One can see a clear trend belonging to the actual track which is in between deviations due to additional hits on the matrix. Since such hits have the same ToA-value as the actual track, they are most likely reflected and scattered photons from the inside and the outside of the scintillator. They do not belong to the track but affect the reconstruction. Therefore, it was necessary to "clean up" the frame before reconstruction.

For this purpose, we calculated the center position for each cluster of adjacent triggered pixels (one photon detection event) and determined the number of neighboring photon detection events within a chosen radius. After choosing a particular radius, clusters are removed from the frame if the number of neighbors within that radius was below a chosen threshold value. A choice of 30 pixels for the radius

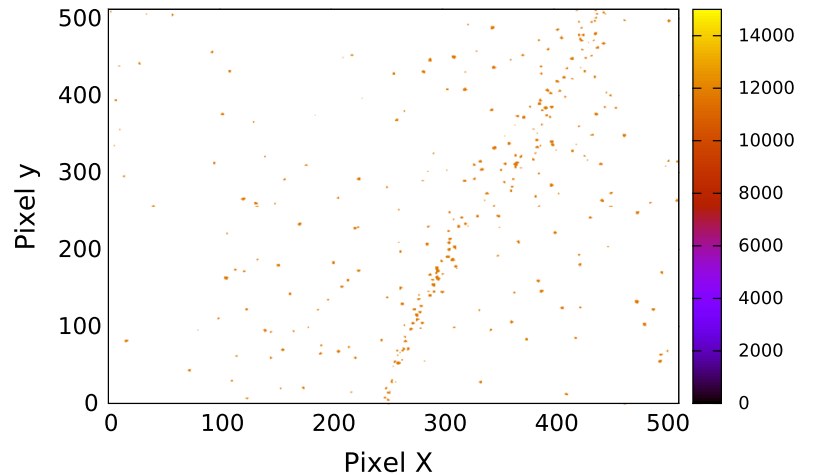

(a)

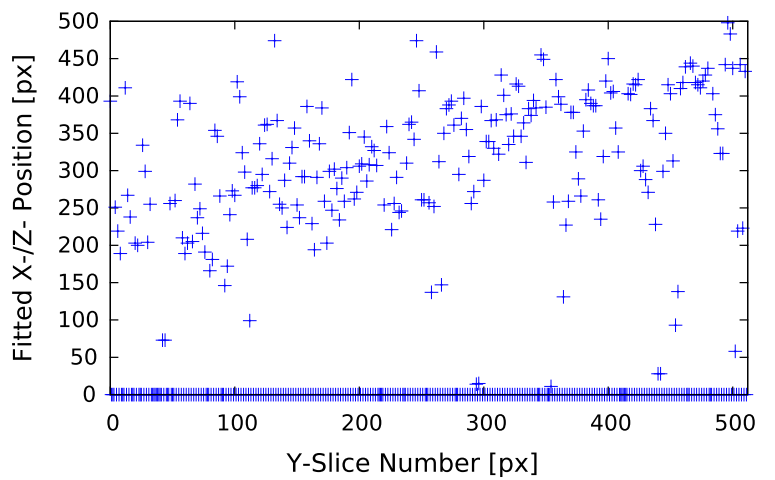

(b)

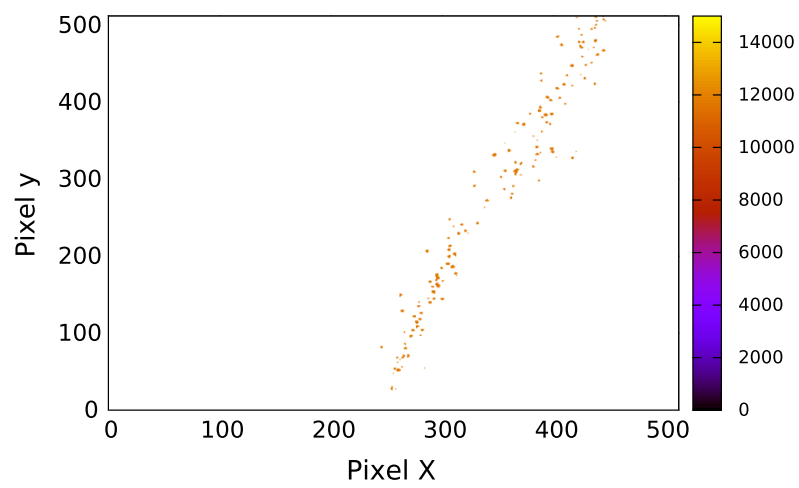

(c)

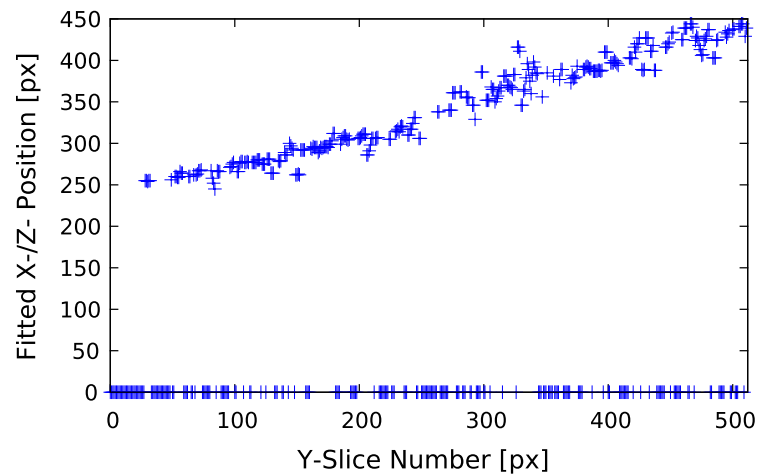

(d)

Fig. 11 Examples of images (a, c) and the fitted $x-/ z$-position for every $y$ slice before (b) and after (d) frame cleanup. The color bar indicates the ToA measured in each triggered pixel 
and of eight neighbors for the threshold value produced reasonable and reliable results. One exemplary frame is shown before and after cleanup in Fig. 11a, c with the $y$-slices (Fig. 11b, d), respectively. One can see that by this procedure the large deviations from the main line are strongly reduced.

The reconstruction of the three-dimensional trajectories was performed by correlating the $y$-slices according to their $y$ value. Every point in the trajectory is referred to as the number of the $y$-slice, the calculated $x$-position in that slice in the $x y$ plane and the calculated $z$-position in that slice in the $z y$ plane. As stated above, we did not take an image of the two projections illuminated with a laser that could provide us with a precise relation between the $x y$ - and $z y$-planes. However, in our case of straight tracks a mismatch of the $y$ coordinate in the $x y$ - and $z y$-planes causes only a linear shift of the complete track in space and therefore does not affect our results on the resolution.

\section{Results}

Two typical reconstructed tracks are shown in Fig. 12. As expected one can see straight lines as trajectories. The main reason for the deviations from the straight line or "broadening" is due to the fact that electrons do not always propagate through the focal plane of the imaging system and therefore the trajectory is blurred. Depending on the distance from the focal plane the blurring affects the achievable position resolution. The second limitation to the position resolution is the resolution of the HPD which is governed by the pixel pitch of the ASIC and the distance between the photocathode and the MCP. The intrinsic position resolution of the HPD in the ToA mode is about $100 \mu \mathrm{m}$, but the width of the blurring circle can range from 0 to several $\mathrm{mm}$, depending on the distance from the focal plane.

Before assessing the position resolution, we evaluated the Gaussian width obtained from the Gaussian fits to the slices. This is an estimate for how close two tracks can be next to each other to be distinguishable. This is an important feature since our final goal is to measure curly tracks of low energetic electrons where different parts of the track can be close to each other. The distribution of the Gaussian widths for our selected data (26 events) is shown in Fig. 13. ${ }^{5}$

In order to determine the position resolution, we fitted a straight line to our reconstructed trajectories and considered the distribution of residuals (deviations of the data from the fitted line) in each point in $x$-direction and $z$-direction. As

\footnotetext{
5 The histogram contains only $8.6 \%$ of the slices because slices that had less then four entries are not included in the histogram. Such slices contain usual only one photon cluster (three pixels in a row) or a part of it (one pixel) and therefore no meaningful fit could be performed.
}

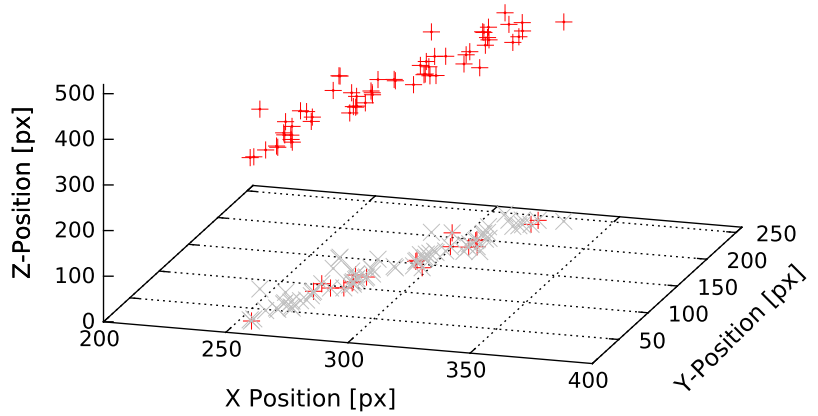

(a)

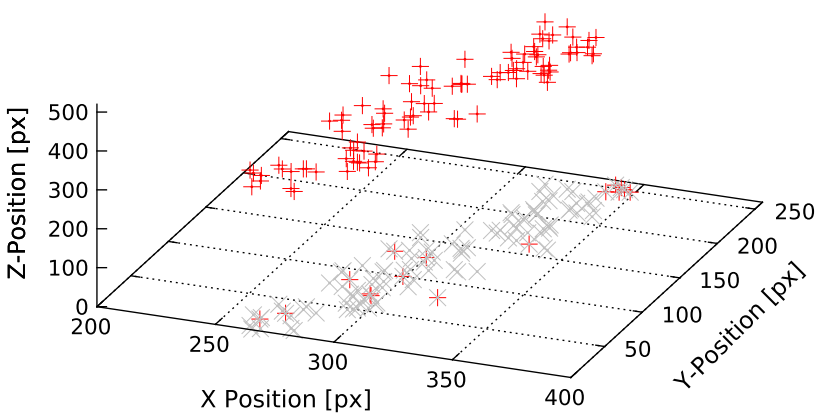

(b)

Fig. 12 Two examples of reconstructed three-dimensional electron trajectories. The red crosses are the reconstructed photon positions of the track. The gray crosses represent the projection of the track on the bottom plane for better visibility. The red crosses on the $x y$-plane are $x y$-slices that had no matching $z y$-slices

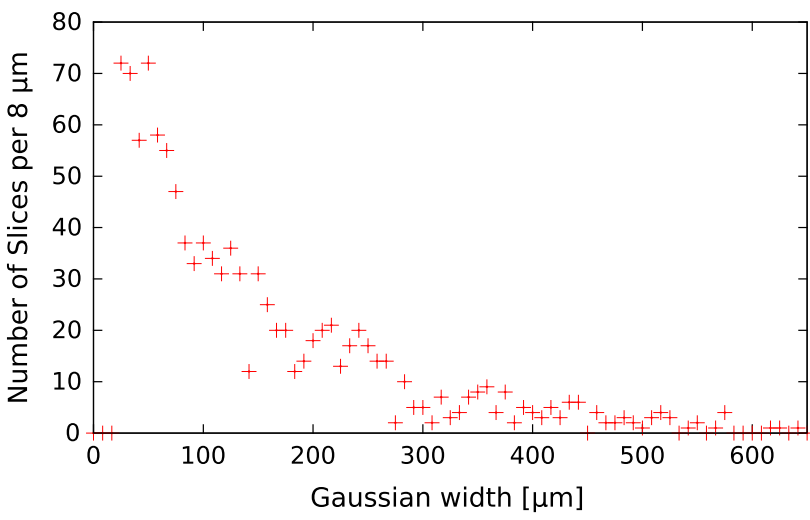

Fig. 13 The distribution of the Gaussian width for the slices of the selected 26 events. The distribution contains only slices which had more than four entries ( $8.6 \%$ of all slices)

the fitted line has very small fit uncertainties due to the large number of points in the track, it can be regarded as the actual track. Hence, the deviations give us an estimate for how close to the actual track a reconstructed point is and how much the positions of the detected photons scatter around the actual track. In Fig. 14 the distributions of residuals in $x$-direction and $z$-direction are shown.

The histogram has a similar shape for both directions. For $z$-direction the width of the distribution seems to be slightly 


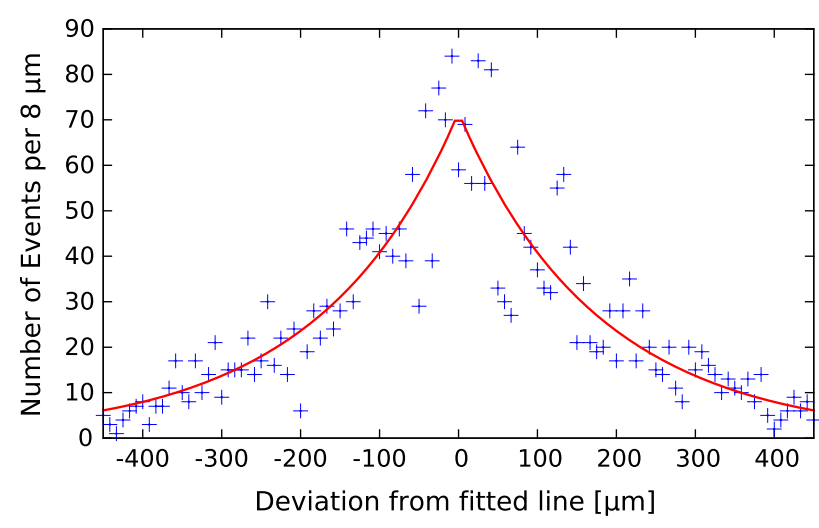

(a)

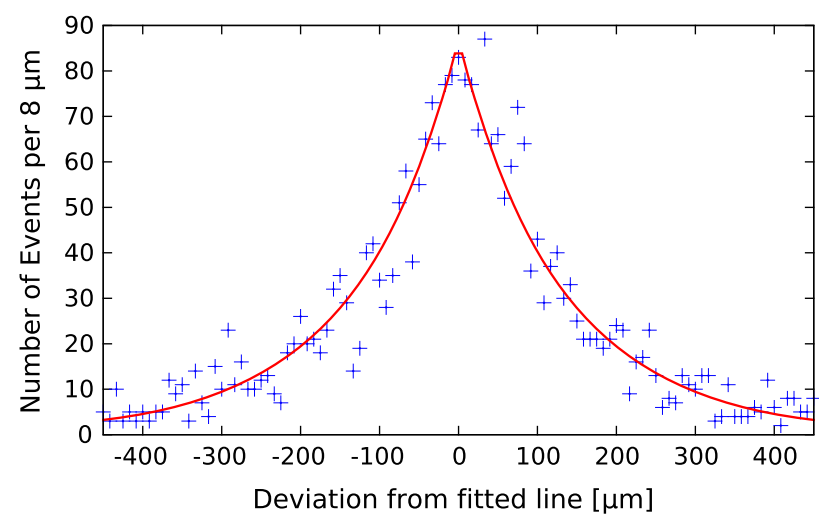

(b)

Fig. 14 The distribution of the deviations from the actual track in $x$ and $z$-directions. The fit function is given in the text. The resulting fit values are $a=71.8 \pm 4.7, b=0.98 \pm 0.11, c=(179.5 \pm 16.8) \mu \mathrm{m}$ ( $x$ direction); $a=87.1 \pm 3.8, b=0.98 \pm 0.07, c=(131.1 \pm 8.5) \mu \mathrm{m}$ (z-direction)

smaller. The fitted distribution function as shown in the plots has the following form:

$f(x)=a \cdot \exp \left(-\left(\frac{x}{c}\right)^{b}\right)$.

We obtained this function from a toy Monte Carlo simulation where the blurring effects of the optical setup are taken into account in the following manner: first, we randomly chose a start position for a photon within an interval of $\Delta d$ around the focal plane according to a uniform distribution. A uniform distribution is chosen since the electron flux can be regarded as homogeneous over the area of the scintillator. Depending on the distance from the focal plane, photons from one point will not end up in one point on the screen but in a blur circle whose diameter can be calculated as

$$
\sigma_{b}=f_{\mathrm{L}} \cdot d_{\mathrm{L}} \cdot \frac{1}{\left(1+\sqrt{1+\left(\frac{\Delta d}{g}\right)^{2}}\right) \cdot \Delta d}+f_{\mathrm{L}} .
$$

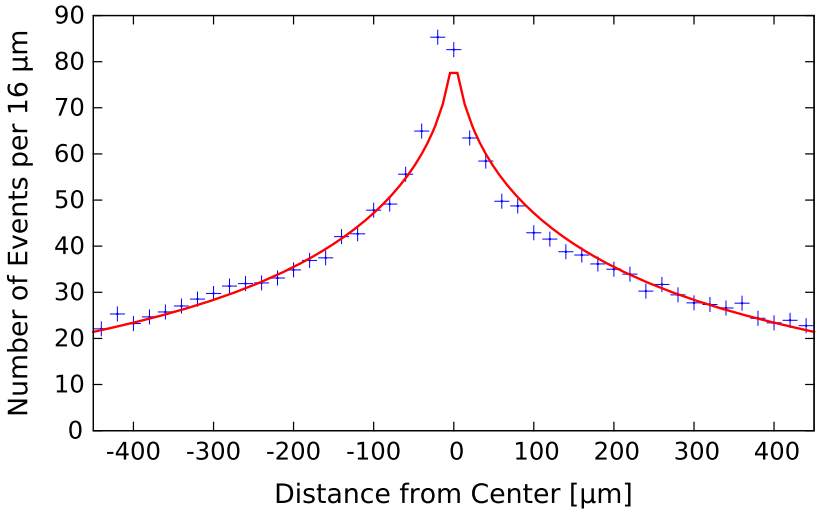

Fig. 15 The distribution obtained from a Monte Carlo simulation for $\Delta d=0.4 \mathrm{~mm}$ in the same range as the experimental data. The data is scaled to the experimental data by the peak height. The fit function is given in the text. The resulting fit values are $a=86.5 \pm 3.2, b=$ $0.55 \pm 0.04, c=(246.3 \pm 19) \mu \mathrm{m}$

Here $f_{\mathrm{L}}$ denotes the focal distance of the lens and $d_{\mathrm{L}}$ its diameter. $g$ is the object distance, i.e. the distance of the focal plane to the lens and $\Delta d$ is the approximate distance of the photon from the focal plane. The formula can be derived from considerations of geometrical optics as can be found in [22], often used in photography. Then we calculated the diameter of the blur circle $\sigma_{\mathrm{b}}$ according to Eq. 2, depending on the distance $\Delta d$ from the focal plane. In the final step, the position of the photon on the screen is chosen randomly according to a Gaussian distribution with $\sigma_{\mathrm{b}}$ as its width. We simulated $10^{5}$ events, which gives a distribution as shown in Fig. 15. The function given in Eq. 1 was obtained as an empirical fit to this distribution.

Although the correspondence of this distribution (Fig. 15) and the result of the experiment is not perfect, the simulation shows that the shape of the distribution is not a Gaussian but can be described by a distribution as given by Eq. 1. We think that this discrepancy is a result of the frame selection and cleaning where tracks which are too blurred are rejected due to the Hough transform and photons are removed which are too much off the tracks. This leads to lower tails in the distribution obtained with experimental data.

The FWHM of the fitted distribution function was in good agreement for simulation and experiment, when we chose $\Delta d$ from $0.25-0.6 \mathrm{~mm}$ as the interval around the focal plane in the simulation. Hence, we might conclude that our frame selection leads to a selection of tracks within this interval around the optical plane. The FHWM of the distribution function (Eq. 1) fitted to the experimental data was $248 \mu \mathrm{m}$ for the $x$-direction and $170 \mu \mathrm{m}$ for $z$-direction. This value can be understood as the position resolution that we obtained with our setup for tracks in a region of roughly $0.25-0.6 \mathrm{~mm}$ around the focal plane. 


\section{Conclusions and outlook}

We have demonstrated that it is possible to reconstruct threedimensional trajectories of particle propagation through a scintillator by imaging two projections of the track on a pixelated single photon detector (the HPD). With our simple optical setup, which consisted of two mirrors and two lenses, and which had an optical magnification of 3.3, we could achieve a resolution of 248 and $170 \mu \mathrm{m}$ in $x$ - and $z$-direction, respectively.

The resolution of the method presented here is mainly limited by the optics used for collecting the scintillation light. However, the main focus was a first "proof-of-principle" demonstration and therefore we concentrated on the easiest possible setup which allowed sufficient light collection efficiency for track imaging, i.e. placing the lens as close to the scintillator as possible. For practical experiments where larger volumes should be imaged a higher depth of focus could be realized with larger lenses and a longer image distance between scintillator and lens at the cost of light collection efficiency. If a high light collection efficiency is required for a good energy resolution, photomultipliers can be placed at the other sides of the scintillator for this purpose.

A possible future application could be the search for neutrinoless double beta decay. In this case high resolution particle tracking is a valuable tool to identify background events and enhance the significance of the observation. Another possible application could be high-energy single photon Compton imaging where particle tracking could be used to determine the momentum direction of the Compton scattered electron.

An additional application could be beam profile monitoring at particle accelerators: high-energetic particles excite the rest gas in the beam pipes which scintillates. An imaging of this beam profile from multiple directions could be useful for beam tuning.

Acknowledgments We cordially thank the Medipix collaboration for the development of the HPD that we have used. This work was carried out within the Medipix collaboration. We would like to thank Ralf Diener and Samuel Ghazaryan from DESY for their support at the T-22
Testbeam line. Also, we would like to thank Dirk Wiedner from the MuPix Group for a share of their beam-time. We would like to thank Felix Just and Andrea Cavanna for their support. Also, we would like to thank Simon Filippov for valuable discussions. We thank the International Max Planck Research School (IMPRS) for supporting M. Filipenko and the Deutsche Forschungsgemeinschaft DFG for supporting T. Gleixner.

Open Access This article is distributed under the terms of the Creative Commons Attribution License which permits any use, distribution, and reproduction in any medium, provided the original author(s) and the source are credited.

Funded by $\mathrm{SCOAP}^{3}$ / License Version CC BY 4.0.

\section{References}

1. W.B. Fretter, Ann. Rev. Nucl. Sci. 5, 145-178 (1955)

2. C.D. Anderson, Phys. Rev. 43, 491-494 (1933)

3. C. Charpak, F. Sauli, Nucl. Instr. Meth. 162, 405-428 (1979)

4. H.J. Hilke, Rep. Prog. Phys. 73, 116201 (2010)

5. L.M. Montaño, J. Phys. Conf. Ser. 18, 368 (2005)

6. P. Collins et al., Nucl. Instr. Method A 636, S185 (2011)

7. K. Akiba et al., Nucl. Instr. Method A 661, 31-49 (2012)

8. M. Campbell, Nucl. Instr. Method A 633, 1-10 (2011)

9. D.D. Ferdinando, Radiat. Meas. 44, 840-845 (2009)

10. A. Gallas, Phys. Proc. 37, 151-163 (2012)

11. S.M. Bilenky, C. Giunti, arXiv:1203.5250 (2012)

12. The EXO-200 Collaboration, Nature 510, 229-234 (2014)

13. M. Schumann, arXiv: $1310.5217 \mathrm{v} 2$ (2013)

14. M. Filipenko et al., Eur. Phys. J. C 73(73), 2374 (2013)

15. T. Michel et al., AHEP 2013, 105318 (2013)

16. V. Álvarez et al., JINST 8, P09011 (2013)

17. E. Aprile, T. Doke, Rev. Mod. Phys. 82, 2053-2097 (2010)

18. J. Vallerga et al., JINST 9, C05055 (2014)

19. M. Platkevic et al., Nucl. Instr. Method A 591, 254f (2012)

20. D. Turecek et al., JINST 6, C01046 (2011)

21. X. Llopart et al., Nucl. Instr. Method A 581, 485-494 (2007)

22. H.M. Merklinger, (Seaboard Printing Limited, Bedford, 1993). ISBN 0-9695025-2-4

23. R.O. Duda, P.E. Hart, Comm. ACM 15, 11-15 (1972) 